\title{
Correction: DUB3 and USP7 de-ubiquitinating enzymes control replication inhibitor Geminin: molecular characterization and associations with breast cancer
}

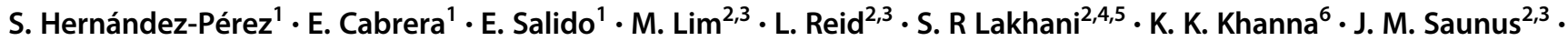 \\ R. Freire ${ }^{1}$
}

Published online: 8 May 2019

(c) Springer Nature Limited 2019

Correction to: Oncogene 36, 4802-4809 (17 August 2017) https://doi.org/10.1038/onc.2017.21 published online 13 March 2017.

The original version of this Article contained an error in the Acknowledgements section.

The final sentence of the Acknowledgements should be as follows: This work was supported by grants from Instituto de Salud Carlos III (BA15/00092), Spanish Ministry of Economy and Competitiveness/EU-ERDF (SAF201680626-R, SAF2013-49149-R, BFU2014-51672-REDC), Fundación CajaCanarias (AP2015/008) to RF, and the Australian National Health and Medical Research (NHMRC program grant to SRL and KKK (APP1017028).

This has not been corrected in the PDF or HTML of this article.

These authors contributed equally: S. Hernández-Pérez, E. Cabrera

\author{
J. M. Saunus \\ j.saunus@uq.edu.au \\ rfreire@ull.edu.es
}

$\triangle$ R. Freire

1 Unidad de Investigación, Hospital Universitario de Canarias, Instituto de Tecnologías Biomédicas, La Laguna, Spain

2 The University of Queensland, UQ Centre for Clinical Research, Herston, QLD, Australia

3 QIMR Berghofer Medical Research Institute, Herston, QLD, Australia

4 Pathology Queensland, The Royal Brisbane and Women's Hospital, Herston, QLD, Australia

5 The University of Queensland, School of Medicine, Herston, QLD, Australia

6 Signal Transduction Laboratory, QIMR Berghofer Institute of Medical Research, Brisbane, QLD, Australia 